\title{
Intervenir avec des masseurs-kinésithérapeutes : le développement en référence
}

Intervening with physiotherapists: development as a reference

\section{Stéphane Balas}

\section{OpenEdition}

\section{Journals}

Édition électronique

URL : http://journals.openedition.org/activites/584

DOI : 10.4000/activites.584

ISSN : 1765-2723

Éditeur

ARPACT - Association Recherches et Pratiques sur les ACTivités

Référence électronique

Stéphane Balas, «Intervenir avec des masseurs-kinésithérapeutes : le développement en référence », Activités [En ligne], 10-1 | Avril 2013, mis en ligne le 15 avril 2013, consulté le 19 avril 2019. URL : http:// journals.openedition.org/activites/584; DOI : 10.4000/activites.584

Activités est mis à disposition selon les termes de la licence Creative Commons Attribution - Pas d'Utilisation Commerciale - Pas de Modification 4.0 International. 


\title{
Intervenir avec des masseurs-kinésithérapeutes : le développement en référence
}

\author{
Stéphane Balas \\ Centre de Recherche sur le Travail et le Développement (EA 4132) \\ Équipe « clinique de l'activité »- CNAM, 62, rue Maurice Thorez, 92000 Nanterre stephane.balas@ wanadoo.fr
}

\begin{abstract}
Intervening with physiotherapists: development as a reference. The design of a profession's standard frame of reference, for training or management purposes, has an effect on the activity of the persons involved in the process. Once completed, however, it becomes a standardizing document, a document which impedes the dynamics of the activity. With a group of physiotherapists, we conducted a clinical intervention that aimed to identify issues in their profession which are unresolved and which we consider as offering a possible access to the "real of the activity". Some of these unresolved issues are permanent and are particularly characteristic of the profession: these are the dilemmas that present professionals, when practicing, with impossible choices between two or more unsatisfactory solutions. Faced with these unsolvable obstacles, yet nevertheless obliged to act, these operators develop and capitalize on their experience. On this basis, our clinical intervention with physiotherapists allowed us to design a standard frame of reference which supports dilemma and acquired knowledge, which, through use, encourages renewed dialogue between professionals. Its clinical efficacy would seem to be explained by the presence of a "proto-referential", a generic instrument of pre-organization implicit in the situations encountered, which the "referentialization" process enables us to rediscover and rediscuss. The artificial setting of clinical intervention thus allows us to reconsider the ecological framework underlying the profession.
\end{abstract}

\section{KEYWORDS}

Activity, Physiotherapists, standard frame of reference, clinical, dilemma

Concevoir un référentiel descriptif de métier, à des fins de formation professionnelle ou de gestion des ressources humaines, en collaboration avec les opérateurs concernés, a un effet sur ces personnes et sur leurs activités. Ce simple constat est le point de départ d'une réflexion, puis d'une action, avec un collectif de masseurs-kinésithérapeutes travaillant dans un centre de rééducation. Nous avons pu, à travers une activité de conception (Olry, \& Vidal-Gomel, 2011) de référentiel de leur métier, expérimenter avec un groupe de professionnels de la kinésithérapie, l'effet transformateur que ce processus pouvait avoir sur leur travail, mais aussi envisager les conditions d'une propagation de cet effet à l'ensemble du métier. Au-delà, nous avons pu percevoir comment la conception d'un référentiel impose toujours de composer entre une visée descriptive qui norme un métier, mais l'arrête, et un objectif transformateur qui maintient le métier en mouvement, mais risque de ne plus 
permettre au référentiel de «faire référence » (Chauvigné, 2010). Cette tension fait écho, en le parant de formes nouvelles, au débat historique des intervenants spécialistes du travail tiraillés, dans l'action, entre une compréhension experte du travail, productrice de connaissances dont on peut parfois douter de l'authenticité, et un effet transformateur du milieu étudié, plus ou moins assumé (Clot, 2008a), mais qui, finalement, n'explique rien.

La voie que nous choisissons est une troisième voie, celle du développement. La « clinique de l'activité » (Clot, 1995) dans laquelle nous nous inscrivons postule qu'il faut " développer pour comprendre avant de parfois comprendre pour développer» (Clot, 2008b). Pour notre part, nous espérons «développer l'activité pour référentialiser le métier avant de pouvoir référentialiser le métier pour le développer ». Ce point de vue est peu fréquent dans le domaine de la conception des référentiels, où le passage de l'activité au référentiel est principalement vu comme un processus extractif et non développemental (Prot, 2007).

Dans un premier temps, nous reviendrons sur une intervention au long cours, conduite avec un groupe de masseurs-kinésithérapeutes, travaillant dans un centre de rééducation (Balas, 2011a). Nous expliquerons les enjeux, pour ces professionnels, à s'engager dans une démarche de co-analyse de leur situation professionnelle commune et sur les effets de cette analyse au plan local, mais aussi sur ceux espérés en direction du métier en général.

Nous focaliserons ensuite notre attention sur les obstacles conceptuels qui jalonnent l'intervention d'un clinicien qui cherche à concevoir un référentiel descriptif de métier fondé sur l'activité. On peut, en effet, souvent constater qu'une activité décrite dans un référentiel classique ${ }^{1}$ est dévitalisée, dépersonnalisée. Nous souhaitons, quant à nous, construire des référentiels qui soient des moyens de relance des dialogues professionnels. Nous montrerons qu'une voie possible pour favoriser cette relance dialogique est alors de tenter de saisir dans les référentiels ce qui, dans le travail, demeure irrésolu et qui reste donc «à discuter » entre professionnels.

Cette proposition a été expérimentée avec différents groupes de professionnels du métier de masseur-kinésithérapeute (Balas, 2011b), avec la conception de référentiels en «dilemmes historiques de métier» (Prot, 2011) et en «acquis d'expérience», comme nous avons nommé ces éléments constitutifs. Les premiers sont des obstacles, qui divisent l'opérateur entre deux solutions opposées également insatisfaisantes. Ils sont communs aux professionnels d'un métier et caractéristiques de son exercice. Ils appartiennent au patrimoine générique (Clot, \& Faïta, 2000) de ce métier et peuvent être repérés dans les dialogues entre professionnels (Balas, 2011b), comme nous le présenterons. Les acquis d'expérience sont les ressources, là aussi génériques, qui permettent aux professionnels expérimentés d'agir, malgré les contradictions que lui oppose un dilemme. Ces acquis sont de nature matérielle, technique, relationnelle, corporelle... On peut dire que le processus de professionnalisation (Sorel, \& Wittorski, 2005) consiste, pour un jeune professionnel, à se confronter, dans des «répétitions sans répétition»(Bernstein, 1996), aux dilemmes du métier et à disposer, peu à peu, du répertoire de ressources patrimoniales qu'il recrute et mobilise pour y faire face. Ainsi, un référentiel qui répertorie les dilemmes du métier et les acquis pour y faire face, permet de concilier deux rôles, a priori, contradictoires : fixer une norme, mais aussi disposer d'un outil de relance des dialogues entre pairs puisque, par nature, un dilemme et la meilleure façon d'y faire face, en s'appuyant sur des ressources diverses, sont des éléments éminemment discutables.

L'activité professionnelle ainsi débattue ne «sort pas indemne» (Clot, 2008b) d'un tel processus. Elle est transformée et enrichie par les points de vue divergents et nous pouvons alors faire l'hypothèse qu'un dispositif de référentialisation de l'activité professionnelle, conduit avec un collectif, peut constituer une technique d'intervention particulièrement

1 Comme on en rencontre par exemple dans les référentiels d'activités professionnelles (RAP) des diplômes professionnels de l'éducation nationale (Maillard, 2008). 
favorable au développement de l'activité concernée, mais aussi du pouvoir d'agir (Clot, 2008a) des professionnels qui exercent le métier. C'est ce que nous chercherons à illustrer, dans une troisième partie, à partir de la présentation d'un repérage de dilemme dans un extrait de dialogue entre professionnels. Cela nous conduira ensuite à exposer le prototype de référentiel construit avec ces professionnels, à partir du repérage systématique de dilemmes et d'acquis des masseurs-kinésithérapeutes.

Nous conclurons nos propos en constatant que si ce cadre d'intervention est artificiel, au sens où il est construit, de manière volontaire, par l'action de l'intervenant auprès d'un collectif, il semble que son efficacité peut trouver son origine dans sa proximité avec un processus, écologique celui-ci, qui poussent les professionnels, dans le travail, à se doter d'une catégorisation a priori, la plupart du temps implicite, des situations affrontées et que nous pouvons comparer à un "proto-référentiel ». Cette manière d'appréhender le réel, en anticipant sa réponse par une identification de la «classe de situations » (Pastré, 2011) rencontrée, permet aux professionnels des gains de temps et d'efficacité. Nous évoquerons comment nous pouvons soupçonner la présence de ce proto-référentiel, propre au métier, à travers certaines locutions employées de manière répétitive par les professionnels, dans des extraits de dialogues d'autoconfrontations en cours d'analyse, telles que «ça dépend » ou encore « des fois ».

\section{1.- Le travail des masseurs-kinésithérapeutes comme objet}

Les masseurs-kinésithérapeutes travaillant dans un centre de rééducation en région parisienne ont accepté de s'engager avec nous dans une activité de co-analyse de leur travail à visée descriptive. Pour comprendre cet engagement qui s'est poursuivi sur plus d'un an, il nous faut présenter les conditions de cette rencontre entre un groupe de professionnels et un intervenant clinicien. Nous exposerons aussi les multiples bouleversements qui touchent actuellement le métier de masseur-kinésithérapeute et qui expliquent sans doute la volonté de ces professionnels de s'engager dans une telle démarche.

Dans le cadre d'un travail de recherche doctorale, nous avons sollicité des professionnels de ce secteur paramédical via un centre de formation qui invite régulièrement des masseurskinésithérapeutes expérimentés, à donner des cours ou participer à des jurys. Parmi les professionnels rencontrés, à qui nous avions exposé notre projet d'intervention et de recherche, un responsable du centre cité précédemment s'est déclaré très intéressé que nous intervenions avec les membres de l'équipe qu'il coordonnait. Il était entendu, dès ce moment, que nous souhaitions construire une action de coanalyse du travail à des fins descriptives, afin d'expérimenter une conception de référentiel. Nous avions également précisé que les professionnels devraient être volontaires et libres de s'engager ou non dans la démarche ${ }^{2}$.

Nous avons alors entrepris une action faite d'une longue période d'observation, réciproque, point de départ de la méthodologie «clinique de l'activité » choisie et qui a permis de vérifier l'engagement des professionnels à participer. Cette phase d'observation est importante, en effet, car «la méthodologie est d'abord, dans l'intervention concrète en clinique de l'activité, la construction avec ceux qui en ont fait la demande d'un renversement [...] La méthodologie est à l'œuvre quand les professionnels concernés sont engagés euxmêmes dans des activités d'observation et d'interprétation de leur propre situation » (Clot, 2008a, p. 31).

Après plusieurs mois de visites au centre, d'entretiens avec les professionnels, de questions réciproques - de notre côté sur ce que nous observions et ne parvenions pas ou feignions de

2 Il était aussi entendu que ce professionnel cadre, responsable de cette équipe de masseurs-kinésithérapeutes, ne pourrait participer directement à la co-analyse, puisque celle-ci ne peut se réaliser qu'entre pairs. 
ne pas comprendre, et, du leur, sur les mobiles de nos visites -, nous avons proposé une réunion collective afin de mettre en place un processus d'autoconfrontation croisée (Clot, Faïta, Fernandez, \& Scheller, 2001). Le cadre de cette méthode prévoit que le collectif associé à la démarche participe au choix des séquences qui seront filmées et qui serviront de support aux entretiens. Il est aussi nécessaire que des volontaires émergent du collectif, deux au minimum et parfois trois ou plus.

Dans le cas présent, deux professionnels, Hervé et Bertrand, ont accepté que leur activité soit filmée. En revanche, le groupe, composé d'une dizaine de professionnels, a renoncé à définir une séquence précise de leur travail, comme la méthode le prévoit, et ce pour plusieurs raisons. Avant de voir ces raisons, précisons rapidement que cet «écart » à la méthode ne nous a pas semblé dommageable pour l'analyse ultérieure. Loin du fétichisme de la méthode, nous considérons celle-ci comme un moyen technique et non comme un dogme. Clot, suivant ainsi les propositions soutenues par Vygotski (1925/2005, p. 43) d'exercer un regard indirect sur l'activité, propose d'organiser les conditions favorables à la «dispute professionnelle » (2008a). Celle-ci ne peut se développer que sur la base des traces du travail réalisé, traces mémorielles dans l'instruction au sosie, traces vidéo pour les autoconfrontations utilisées ici. L'essentiel était donc que les professionnels puissent disposer d'un cadre réglé de mise en débat de leurs activités de travail, en s'appuyant sur des traces de celles-ci. Dans certains métiers très répétitifs, les séquences à filmer sont assez facilement repérables. Dans les métiers marqués par des actions plus diverses, comme c'est le cas dans le soin, les collectifs de professionnels oscillent souvent, dans leur choix, entre une volonté de «tout» montrer, ce qui est évidemment impossible, et des propositions réduites à quelques situations jugées « critiques ».

Nous avons choisi, dans cette intervention, de ne pas intervenir de manière directive dans cette phase, postulant que ces traces, qui relève du réalisé, doivent surtout avoir du sens pour les professionnels, afin de favoriser la découverte d'autres possibles de l'action et ainsi tendre vers le réel de l'activité. Les masseurs-kinésithérapeutes ont ainsi choisi de ne pas choisir.

La première raison de la non-définition d'une séquence est que leurs activités, dans ce centre, sont «entremêlées » les unes aux autres. Un professionnel travaille souvent avec plusieurs patients en même temps. Pendant qu'il en masse ou mobilise un ${ }^{3}$, il contrôle, visuellement, des exercices réalisés par un autre ce qui rend toute circonscription de séquence très difficile. De plus, nous avions observé pendant la phase précédente, une omniprésence de l'humour entre professionnels, mais aussi dans les relations avec les patients. Les masseurs-kinésithérapeutes associés à cette démarche ne voulaient pas, par une détermination de séquences prédéfinies, «aseptiser » l'activité et interdire la survenue du rire ou le «thêatraliser ». On connait les divers rôles que l'on peut prêter au rire dans le travail (Bournel-Bosson, 2005), dont une fonction défensive. La survenue du rire signale alors souvent au clinicien, un implicite particulièrement utile à faire émerger, par un travail d'analyse, avec les professionnels.

Nous avons pu aussi relier ce choix de ne pas prédéfinir de séquences à filmer, avec la volonté explicite des professionnels de s'approcher d'une analyse objective et authentique de leurs activités. En effet, comme nous avons pu le développer par ailleurs (Balas, 2011b), leur métier connaît aujourd'hui, comme à l'époque, de multiples tentatives de référentialisation, concurrentes, et qui laissent les professionnels dans un sentiment de ne pas être acteur de la définition de leur métier et de son avenir. Si cette inflation de référentiels laisse imaginer que chacun ne peut plus faire référence que pour le cercle de ses concepteurs, les travaux de «réingénierie » du diplôme et de la formation de masseur-kinésithérapeute, conduit actuellement au sein du ministère de la Santé, sont ce qui interroge le plus ces

3 Pour une rééducation articulaire. 
professionnels. La période de démarrage de l'intervention était, de ce point de vue, pleine d'incertitudes face à l'annonce de ces travaux, sans avancées palpables et avec une représentation professionnelle contestée $e^{4}$.

De plus, le métier de masseur-kinésithérapeute, comme tous les métiers de soin, vivait alors une transformation socio-institutionnelle très importante. Sans prétendre à l'exhaustivité nous pouvons citer la réforme de l'hôpital (par exemple le regroupement des hôpitaux au sein de pôles régionaux, la réforme de l'Assistance Publique-Hôpitaux de Paris, AP-HP) et un projet de gestion prévisionnelle des métiers et des compétences porté par la fonction publique hospitalière ${ }^{5}$, l'intégration des études médicales et paramédicales au système de l'enseignement universitaire (dit modèle LMD - Licence, Master et Doctorat), la définition d'un code déontologique pour les masseurs-kinésithérapeutes (décret du 3 novembre 2008) qui constitue le premier acte de l'ordre des masseurs-kinésithérapeutes créé en septembre 2006. Nous pouvons aussi évoquer les divers textes qui influencent directement l'exercice professionnel de ces soignants comme le décret modificatif du 7 août 2004 relatif aux actes professionnels et à l'exercice de la profession de masseur-kinésithérapeute ou celui du 29 juillet 2009 qui définit le bilan kinésithérapique et reconnait au kinésithérapeute une compétence à diagnostiquer une affection et à prescrire un traitement, capacité jusqu'ici réservée au médecin.

Ces bouleversements, dont les professionnels du centre de rééducation se sentaient plus victimes qu'acteurs, entraînaient une forte inquiétude et une volonté, pour certains, de repenser collectivement ce qu'est un bon geste, une bonne technique, un bon soin. En un mot, face à un environnement qui change, ce groupe de professionnels souhaitait retrouver un repère stable à travers la description de ce qui fait d'un masseur-kinésithérapeute, un «bon professionnel».

Notre proposition de produire avec eux une co-analyse de leurs activités, avec un objectif explicite de rédiger un référentiel descriptif de leur métier, a donc été saisie comme une opportunité et l'engagement de ces professionnels a alors été acquis.

Si le cadre d'analyse «clinique de l'activité »(Clot, 1995) mis en place, sur lequel nous allons revenir, a un effet sur l'activité des professionnels qui y sont directement associés, le fait de produire un référentiel descriptif de leur métier, laissait espérer, dès le démarrage de l'intervention, que cet outil produit pourrait ensuite devenir un instrument (Verillon, \& Rabardel, 1995) de propagation des effets de l'intervention sur des milieux professionnels élargis.

Mais revenons d'abord sur la difficulté de concevoir un référentiel descriptif d'un métier à partir d'activités professionnelles, par nature, vivantes et dynamiques.

\section{2.- Analyse du travail et référentialisation}

Dans ce contexte de changement rapidement décrit, les deux professionnels volontaires, Hervé et Bertrand, ont donc accepté d'être filmés, en situation professionnelle, pendant environ une heure chacun. Les images recueillies ont ensuite servi de support à un entretien d'autoconfrontation simple (Clot, et al., 2001) où chacun des professionnels, en présence du clinicien, est amené à visionner et commenter ces traces de son activité. Cette phase déclenche chez ce professionnel un étonnement, entretenu par les questions du clinicien, face

4 Dans ces groupes de réingénierie étaient conviés, pour représenter les professionnels, des instituts de formation et des représentants syndicaux, tous, sans doute, légitimes pour défendre le point de vue de leurs organisations, mais pas toujours jugés aptes à rendre compte du métier quotidien des professionnels.

5 Cette fonction publique s'est dotée d'un répertoire des métiers et d'un arsenal méthodologique permettant de lier en cascade, métiers, activités requises, savoir-faire, connaissances associées aux savoir-faire, ces deux derniers éléments étant constitutifs de la compétence. 
aux écarts entre ce qu'il se voit faire sur le film et ce dont il avait le souvenir, ou qu'il pensait, a posteriori, logique et explicable. Mais, « ce qui compte, c'est ce qu'on n'arrive pas encore à dire du réel de l'activité : "ce difficile à dire" avec quoi on pourrait peut-être faire quelque chose de différent de ce qu'on fait. [...] Dans le genre d'analyse du travail dont il est ici question le "difficile à expliquer" est, pour ainsi dire, le furet du jeu dialogique » (Clot, 2005, p. 66).

Pour Hervé et Bertrand, les deux masseurs-kinésithérapeutes associés à notre démarche, le dialogue s'est poursuivi au cours d'une autoconfrontation croisée où ensemble, et avec le clinicien, ils peuvent visionner les images du collègue et rouvrir les débats de métier, là où l'isolement professionnel les laisse parfois immobiles et indiscutables et ainsi s'expliquer, individuellement et avec le collègue, sur ce difficile à dire. Les controverses qui peuvent se développer entre collègues, sur un geste ou une action, lors de ces dialogues, sont alors des fenêtres ouvertes vers le réel de l'activité, dont Vygotski nous a appris qu'il constitue un volume dont le réalisé n'est que la surface (Clot, 2004, p. 31). En effet, le comportement tel qu'il s'est réalisé «est une infime part de ce qui est possible. L'homme est plein à chaque minute de possibilités non réalisées »(Vygotski, 2003). Et ce réel est bien ce que l'on souhaite approcher, pour en décrire une part.

L'ensemble des entretiens est filmé et donne lieu à une retranscription intégrale des dialogues. Dans notre exemple, ces matériaux ainsi constitués ont eu une double fonction: servir de support pour construire un retour vers le collectif initial dont les deux professionnels sont issus; mais aussi permettre de rédiger un référentiel descriptif de la situation professionnelle des masseurs-kinésithérapeutes du centre de rééducation.

Cette démarche de référentialisation est donc «un processus d'élaboration du référent (articulé autour de ses deux dimensions : générale et situationnelle). La référentialisation consiste à repérer un contexte et à construire, en les fondant sur des données, un corps de références relatif à un objet (ou une situation) par rapport auquel pourront être établis des diagnostics, des projets de formation et des évaluations. La référentialisation veut être une méthode de délimitation d'un ensemble de référents et se distingue en cela du référentiel qui désigne, lui, un produit fini, et plus exactement, une formulation momentanée de la référentialisation » (Figari, 1994, p. 48).

Si le propos de cet auteur est plus orienté vers des questions d'évaluation que de description, dont nous avons cherché, par ailleurs, à montrer la nécessaire différentiation quand on veut concevoir des référentiels (Balas, 2011b), il n'en reste pas moins que deux éléments sont notables. D'abord, Figari distingue le processus du résultat ce qui confirme l'intérêt de tenter de dépasser la nature "finie » du référentiel, comme nous cherchons à le faire, afin de retrouver dans le résultat, les propriétés du processus. D'autre part, il insiste sur le fait que ce référentiel, s'il est un produit fini, ne l'est que momentanément. Nous partageons cette opinion et nous souhaitons même trouver des solutions pour que le référentiel concilie une nature fixiste et un «mouvement perpétuel ».

Nous pensons, en effet, que c'est là une condition pour que les professionnels dont le métier est décrit puissent s'y reconnaitre. En effet, un métier, une activité professionnelle, sont des entités vivantes, et pour que le référentiel devienne un instrument de développement du métier, et non un document uniquement normalisant et parfois sclérosant, il faut parvenir à en faire une description « vivante».

Rabardel évoquant le rapport entre l'artefact et l'instrument, cherche, à partir des travaux de Vygotski, à construire une théorie instrumentale élargie. Selon lui, cette théorie « doit aussi permettre de rendre compte de la façon dont les instruments se constituent pour le sujet dans une intime relation avec les artefacts inscrits dans l'histoire et la culture de la société dans laquelle il vit et comment, en retour, les instruments des sujets comme des collectifs contribuent aux évolutions sociales, culturelles et historiques » (2002, p. 272). C'est bien ainsi que nous imaginons la fonction des référentiels. 
Pour cela, nous avons fait l'hypothèse, dans la continuité de quelques expériences précédentes dans d'autres milieux professionnels (Clot, Litim, Prot, \& Zittoun, 2008 ; Clot, Tomàs, \& Kloetzer, 2009 ; Prot, Ouvrier-Bonnaz, Mezza, Reille-Baudrin, \& Verillon, 2010 ;) que ce qui peut faire référence, en gardant à l'activité son caractère dynamique, est ce que nous désignons comme les « irrésolus » du métier, c'est-à-dire ce qui pose, de manière récurrente, problème à chaque professionnel du métier et qui résiste à se régler. En effet, dans l'histoire d'un métier, dans la confrontation de ceux qui l'exercent avec la diversité des situations qu'offre le réel, les obstacles sont nombreux et une perpétuelle activité de résolution des problèmes existe.

Pourtant, parmi les très nombreux problèmes que doit affronter un professionnel pour travailler, on sait en effet que tout ne peut se prévoir ou se prescrire (Ombredane \& Faverge, 1955), certains sont particulièrement résistants à se traiter et ne peuvent, au mieux, que se gérer dans l'action. Ils sont et demeurent irrésolus. Ces problèmes aux caractéristiques particulières sont les «dilemmes de métier » qui imposent au professionnel, pour agir, de choisir entre deux solutions, voire deux orientations également insatisfaisantes entièrement. Cette bipolarité du dilemme lui confère cette propriété de ne jamais pouvoir se régler et disparaitre aux yeux des professionnels, à l'exception d'évolutions techniques ou organisationnelles majeures ${ }^{6}$. Ce dernier constat permet aussi de rappeler la dimension temporelle de ces dilemmes qui constituent une sorte de patrimoine historique du métier.

Pour agir, alors même qu'il est confronté aux contradictions engendrées par le dilemme, et afin de «supporter» le sentiment d'incomplétude qu'il ressent, le professionnel est alors amené à développer son expérience et à conserver les acquis de celle-ci, sous diverses formes : détournement d'usage d'instruments ${ }^{7}$ techniques ou langagiers, mode relationnel, engagement corporel. Ces acquis d'expérience sont des créations individuelles qui s'appuient sur le genre du métier et y retournent pour y faire patrimoine, eux aussi. Leur développement suit une forme comparable à celui déjà décrit (Clot, \& Faïta, 2000) pour ce qui concerne le couple constitué du genre et du style.

Nous pensons que produire un référentiel descriptif de métier répertoriant les dilemmes historiques et les acquis d'expérience de ce métier permet de disposer d'un outil qui fasse référence, mais qui reste, foncièrement, discutable.

\section{3.- La référentialisation comme technique d'intervention}

La clinique de l'activité, avec laquelle nous avons choisi d'outiller au plan méthodologique notre intervention, vise explicitement à permettre aux professionnels engagés de reprendre la main sur l'histoire de leur métier en développant leur pouvoir d'agir ${ }^{8}$, c'est-à-dire le rayon d'action de leurs activités (Clot, 2008). Cette clinique repose sur des méthodes dites « indirectes » (Vygotski, 1925/2005, 2011) qui permettent de proposer un cadre artificiel

6 Du type robotisation d'une tâche ou encore reconnaissance d'une capacité nouvelle à une catégorie de professionnels (comme c'est le cas pour la prescription kinésithérapique).

7 Ce que l'on désigne par le terme de catachrèse (Clot, \& Gori, 2003).

8 Le terme, et le concept de «pouvoir d'agir », font l'objet d'une discussion scientifique. D'un côté, Rabardel y voit un des effets de l'activité qui se montre, d'un côté «productive » et de l'autre « constructive » (Rabardel, \& Pastré, 2005 ; Samurçay, \& Rabardel, 2004). C'est la dimension constructive de l'activité, séparée de son orientation productive, qui transforme l'individu en « sujet capable », le construit, et lui permet d'augmenter son pouvoir d'agir sur le monde. Clot, quant à lui, conteste cette dichotomie en indiquant que "l'activité n'est cognitive ou émotive qu'au second degré, elle est d'abord mouvement d'appropriation d'un milieu de vie par le sujet, ou amputation de celui-ci » (2008a, p. 6). Ainsi, le pouvoir d'agir est ce qui lui permet de produire de nouveaux objets de l'activité, de nouveaux instruments, de nouveaux publics, de nouvelles institutions du métier qui vont transformer in fine la prescription. En un mot, l'activité est transformatrice pour l'individu, parce qu'elle est transformatrice pour l'objet. L'individu se construit et développe son pourvoir d'agir sur le monde en agissant sur le monde (ibid.). 
favorable à l'instauration de controverses professionnelles dont on connait la valeur développementale (Clot, 2008a ; Clot, et al., 2001).

L'originalité de notre démarche est d'utiliser ce cadre d'action et de recherche pour concevoir un référentiel descriptif de métier. Si la clinique de l'activité puise ses racines dans l'ergonomie francophone et dans la psychopathologie du travail (Simonet, Caroly, \& Clot, 2011), nous avons fait l'hypothèse qu'elle permettrait aussi de contribuer, par son approche de l'analyse du travail, à outiller une démarche à visée didactique et d'ingénierie. Précisons simplement que ce second objectif ne se substitue pas aux objectifs d'action habituels de la clinique de l'activité, mais les complète.

Nous postulons même que l'objectif, explicite et partagé avec le groupe de professionnels, de décrire leurs activités afin de référentialiser leur métier, est une technique d'intervention efficace qui concoure à accompagner le développement du pouvoir d'agir des professionnels, localement. Il permet même de propager, dans des conditions qui restent à confirmer, ces effets transformateurs à des échelles plus vastes, grâce à la nature impersonnelle (Clot, 2008a) du référentiel produit.

Pour cela, il faut cependant se conformer à une condition : il faut produire, comme nous l'avons déjà indiqué, un référentiel qui reste, par nature, discutable. Sinon, les effets transformateurs liés au processus de référentialisation, quand ils associent des professionnels du métier décrit, s'éteignent à proportion du caractère normalisant et fixiste du référentiel produit.

Nous avons choisi, pour favoriser un usage instrumental (c'est-à-dire discutable au sens où l'artefact normalisant devient un instrument dynamogène pour la reprise des dialogues de métier - Rabardel, 2005) du référentiel, par les professionnels concernés, de décrire les irrésolus du métier en répertoriant les dilemmes de métier et les ressources pour y faire face, les acquis d'expérience. Voyons concrètement cette démarche, avec les masseurskinésithérapeutes.

À l'issue d'un entretien d'autoconfrontation croisée, d'une durée de plus d'une heure et demie, où Hervé et Bertrand ont pu débattre, avec passion, de leur métier et en redécouvrir la «polyphonie », c'est-à-dire la pluralité des points de vue qu'il focalise, nous avons réalisé une retranscription intégrale des dialogues.

Sur la base de ces traces, nous avons, avec ces deux professionnels, travaillé à concevoir un «pré-référentiel» en dilemmes et acquis, afin de revenir devant le collectif initial, rendre compte du travail d'analyse produit. Nous avions, dans cette démarche, la volonté de produire un document inachevé, afin de laisser au collectif, sa part dans la conception.

L'objectif était bien que ce référentiel, ainsi constitué dans un processus itératif avec les deux professionnels volontaires, soit un bon moyen pour relancer les dialogues avec les professionnels du collectif initial, et au-delà. La proposition que nous avions faite aux deux professionnels volontaires était que nos formulations initiales de dilemmes et d'acquis, repérés dans l'intervention et l'analyse de leur dialogue, soient conservées en l'état. Ainsi, elles pouvaient être affinées, retravaillées. Leur degré de généricité restait aussi discutable avec le groupe élargi. Les effets de cette intervention d'une durée conséquente ne se sont d'ailleurs pas arrêtés avec l'ultime retour au collectif, en particulier pour Hervé et Bertrand. Ces derniers, plusieurs années après, continuent à entretenir une correspondance avec nous, témoignant de réflexions sur leur métier, de projets... On peut dire qu'ils ont pris au mot notre consigne de garder leur métier discutable. Cette forme «d'acculturation clinique » des professionnels engagés avec nous, déjà constatée lors d'autres interventions (Kostulski, Clot, Litim, \& Plateau, 2011), signe un effet potentiellement développemental pour l'intervention.

Au cours de cette phase de préparation de retour au collectif initial, nous avons aussi pu remarquer que, dans la retranscription de l'autoconfrontation croisée, l'identification d'un dilemme correspondait à des séquences dialogiques très spécifiques. 
Nous avons alors postulé qu'il est possible d'outiller notre démarche en repérant de manière systématique les dilemmes, dans ces dialogues entre professionnels.

Il semble que, pour identifier des dilemmes de métier, nous devions chercher, dans la motricité dialogique (Clot, 2008a ; Tomàs, 2007), non des controverses déployées, mais « des figures et configurations porteuses de sens qui font vivre la signification » (Clot, ibid., p. 117) et qui contiennent :

— Deux points de vue argumentatifs inconciliables,

- Un échange où l'engagement affectif est palpable (hésitations, usages de gestes démonstratifs, utilisation de formules ou de termes anachroniques) à la poursuite d'un « furet dialogique », où les deux professionnels se confrontent au « difficile à expliquer » évoqué plus haut,

— Un partage et non une opposition entre les deux professionnels, à la fin de la séquence.

Dans l'exemple ci-dessous, les termes ${ }^{9}$ du dilemme identifié sont «fonder sa technique sur des preuves scientifiques ou des constats empiriques ».

L'extrait de dialogue analysé comprend vingt-et-un tours de parole ; les deux professionnels y sont désignés par l'initiale de leur prénom (Tableau 1).

L'échange porte sur les images d'Hervé qui apporte un soin de rééducation du genou d'une patiente, suite à une pose de prothèse, en pratiquant une mobilisation de l'avant-pied de cette dernière (Image 1). Il faut préciser, pour mieux comprendre les propos des deux masseurskinésithérapeutes, qu'Hervé poursuit, au moment de l'intervention et à titre personnel, une formation d'ostéopathe, complémentaire à sa formation initiale.

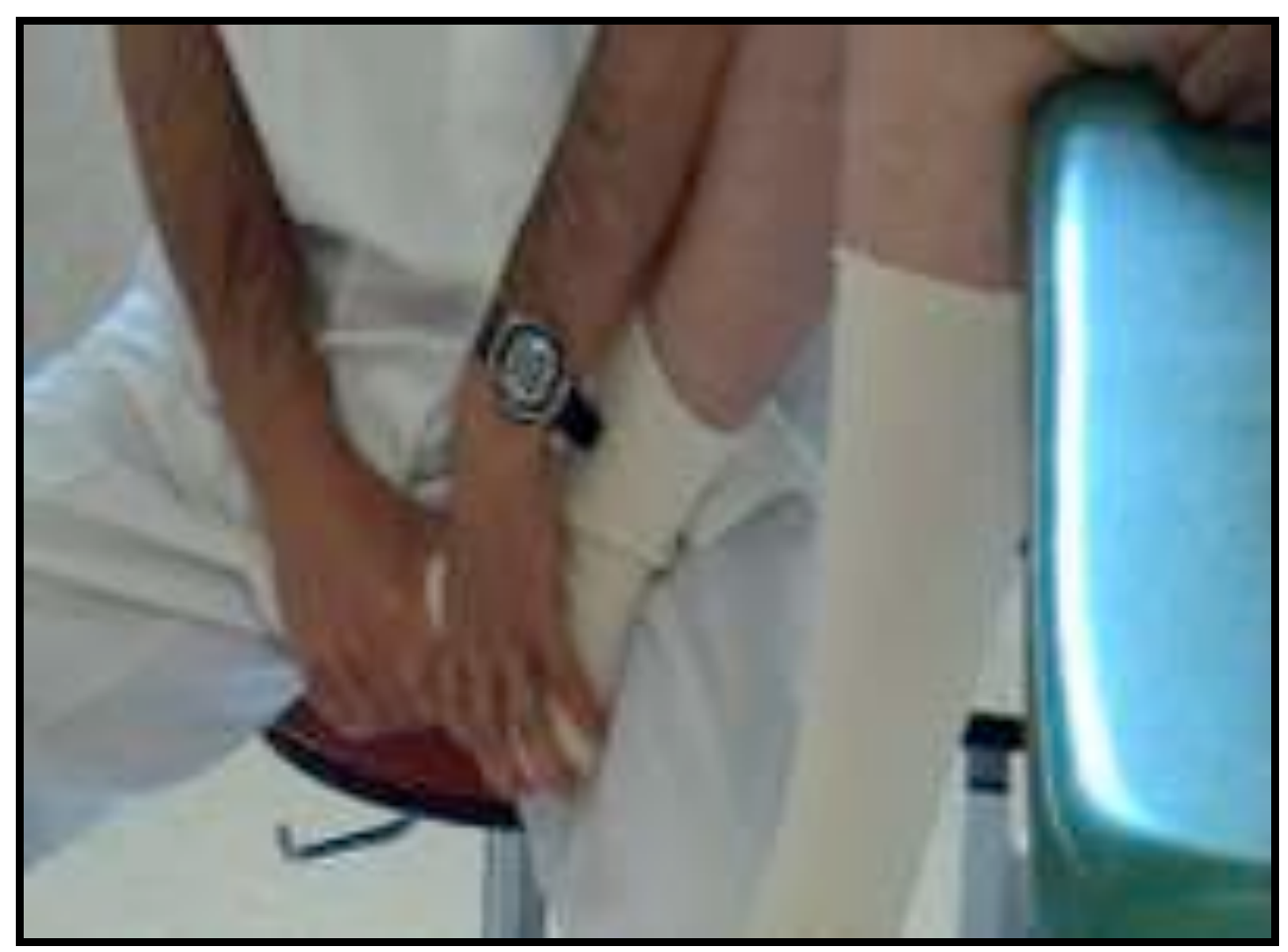

Image $1:$ Hervé mobilise l'avant-pied de la patiente

Image 1: Hervé mobilizes the patient's forefoot

9 Définis par les masseurs-kinésithérapeutes et qui ont fait l'objet de débats passionnés avec les membres du collectif initial. 
225 B. Tu fais quoi, là, en bas ?

226 H. (Rire, prend le chercheur à témoin)

227 B. Tu fais de l'ostéo, là encore, tu refais de l'ostéo, tu t'es lâché, là encore

228 H. Mais, une mobili., une mobilisation d'un scaphoïde, ça va, ça c'est pas, simplement de l'ostéo, (Bertrand : mais pourquoi tu lui fais ?) ça fait partie de la vie. Mais c'est une bonne question

229 B. Pourquoi tu lui fais

230 H. Je ne sais pas, d'après toi, heu non

231 B. Bah, je ne sais pas, là je ne sais pas.

232 H. Tu n'as pas d'idée du tout?

233 B. Non, là, je n'ai pas d'idée du tout. Peut-être la fibula. Moi j'aurais dit, moi j'aurais dit « tu bouges la cheville pour faire bouger la fibula », je me dis ce serait un truc d'ostéo, ou...

234 H. C'est bien parce qu'on en a parlé de ça ensemble

235 B. Moi, je me serais, moi je me serais dit, il bouge la cheville pour activer la fibula qui ferait bouger un peu au niveau du genou. Vu que tu es sur le genou, je me serais dit ça, mais, là, si tu parles de scaphoïde, je ne sais pas

236 H. Non mais ça, c'est, c'est.

237 Ch. C'est la fibula, c'est le péroné ? (les deux : le péroné, ouais) Je traduis pour les non-initiés

238 H. Mais ça, je le faisais avant ma formation

239 B. Bah, tu fais quoi, alors?

240 H. C'est que en fait j'ai remarqué souvent que les genoux que les gens avaient un pied, heu, un pied qu'était rétro... enfin toutes les petites articulation (geste des mains) qui sont dans le pied, notamment entre, heu enfin au niveau du scaphoïde astragale, souvent il y a des gens qui ont (geste des mains), qu'ont des raideurs à ce niveau là, et alors j'ai remarqué que souvent c'était des posi... non mais je ne sais pas si tu l'as remarqué ?

241 B. Je ne sais ce que je vais te dire (Hervé : vas-y). C'est empirique, non ?

242 H. Non, non, ce n'est pas empirique

243 B. (rire) non, parce qu'on a discuté de ça au Mac Do. On a dit que ce qui était empirique devait... Il fallait savoir que c'était empirique... mais une constatation, c'est empirique (rire)... fallait pouvoir le dire que c'était empirique, que c'est une constatation et que ce n'est pas, pas sortir une explication à la (mord moi le nœud)

H. Non, non, mais ce n'est pas simplement, juste parce que je suis allé regarder, je suis tombé dessus par hasard, heu, (Bertrand : mais vas-y). Souvent les gens qui ont des problèmes au niveau du membre inférieur, enfin, au niveau du genou ont l'attitude du pied un peu en varus heu, tu vois? Une sorte de protection du... avec une (Bertrand : mais, après l'opération ?) préposition du... ouais... et tu remarqueras souvent, il y a des gens qui sur le médio pied qui sont, qui sont un peu raides et des fois quand tu, quand tu libères un petit peu ça tu as l'impression, enfin, ce n'est pas une impression que, au niveau de la flexion du genou (geste avec les doigts) tu sens que c'est un petit peu plus (Bertrand : mais c'est) simple à mobiliser

245 B. Mais c'est bien ce qu'on discuté ce midi. Ce midi, juste avant d'arriver, on parlait du, de, de l'empirisme dans la justification des techniques, que quand les gens... que en formation parce justement on discutait de sa formation ostéo sur, sur les gens donnent des explications et des fois faut pas essayer de donner des explications extravagantes quand ils n'ont pas vraiment la vraie réponse, parce que du coup ça décrédibilise le truc. On a le droit de dire que de façon empirique, on a remarqué que ça marchait mieux, ça attend de trouver une explication, mais ça n'empêche pas de le faire, parce que si, si de façon empirique on voit vraiment qu'il y a une vraie efficacité de la technique, c'est d'ailleurs là-dessus que repose la kinésithérapie, parce que pas grand chose n'est prouvé, si ce n'est que de façon empirique on a remarqué que les gens allaient mieux. On n'a pas expliqué encore, il y a plein de chose qu'on n'explique pas... comment ça marche, donc... et on parlait justement de ce côté empirique des choses et que si on remarque quelque chose de flagrant, même s'il n'y a pas de lien immédiat, il y a peut-être un lien quand même, qu'on n'explique pas tout de suite... Et j'essayerai, du coup, Hervé. Même si je n'avais jamais remarqué. Et donc (Hervé : vas-y)

\section{Tableau 1 : Extrait d'autoconfrontation croisée entre Bertrand et Hervé}

\section{Table 1: Extract from a crossed self-confrontation between Bertrand and Hervé}

La technique qu'il pratique dans la séquence filmée, discrète (et surprenante puisque éloignée du genou de la patiente), relève d'une initiative de sa part que Bertrand pointe immédiatement. Ici, les termes de l'échange sont : pratiquer une technique dont l'efficacité est avérée scientifiquement ${ }^{10}$ ou bien pratiquer une technique dont on a constaté,

10 Pour les masseurs-kinésithérapeutes, « scientifiquement » veut dire en laboratoire ou au moins expérimental. 
empiriquement, la valeur.

Dans cet exemple, Hervé tente, dans un premier temps, de se soustraire au regard inquisiteur de son collègue et comme, entre "gens de métier », on ne peut se raconter d'histoire, il finit (en 240) par avouer que son geste repose sur un constat personnel (C'est que en fait j'ai remarqué souvent que les genoux que les gens avaient un pied, heu, un pied qu'était rétro...). Pourtant, quand son collègue lui indique que c'est de l'empirisme, Hervé résiste encore (242: Non, non, ce n'est pas empirique). Alors Bertrand rappelle en 243 le sens du terme empirique (mais une constatation, c'est empirique).

Hervé résiste encore à accepter que son choix de technique avec cette patiente soit qualifié d'empirique. Il se lance dans une série d'explications pour différencier sa pratique de celle qui tiendrait du hasard auquel il assimile l'empirisme. Bertrand conclut en tranchant son propos. (244: les gens donnent des explications et des fois faut pas essayer de donner des explications extravagantes quand ils n'ont pas vraiment la vraie réponse, parce que du coup ça décrédibilise le truc) puis en confirmant l'intérêt d'assumer des choix même si (ça attend de trouver une explication).

La motricité dialogique est singulière dans cet extrait choisi. Dans ces vingt-et-un tours de parole se rejoue l'affrontement de la technique à l'efficacité prouvée face à la technique à l'efficacité éprouvée. Ce qui est remarquable, c'est que les deux professionnels ne campent pas sur une position, mais que, si Hervé se défend de défendre la position de l'empirisme, et assume donc, par défaut, la posture des techniques scientifiques ${ }^{11}$, Bertrand, lui, assume les deux positions puisqu'il pousse Hervé à assumer son empirisme, dont il juge ridicule de se défendre, mais indique aussi qu'en quelque sorte, c'est du scientifique qui n'a pas abouti (ça attend de trouver une explication). Son questionnement, quasiment clinique ${ }^{12}$, mais aussi didactique, amène son collègue, non à venir sur ses positions, mais à assumer ses propres positions empiriques pratiquées, mais presque de façon coupable...

Nous pouvons qualifier cet extrait de dialogue collaboratif maïeutique au sens où la technique de Bertrand consiste bien à interroger Hervé pour lui faire exprimer (accoucher) une vérité qu'il se cache à lui-même.

Si l'on se réfère aux trois indicateurs énoncés précédemment, on peut constater pour cet extrait :

- Que deux points de vue inconciliables s'expriment clairement: «la technique à l'efficacité prouvée scientifiquement» ou «la technique à l'efficacité éprouvée empiriquement »,

— Le « furet dialogique » identifiable dans ce dialogue est le terme «empirique » qui oblige Hervé à s'en défendre, pour finalement l'admettre en fin de séquence, en ne reprenant pas son collègue et en relançant le film à la place de Bertrand qui est sensé «piloter» le visionnage des images d'activités d'Hervé lors de cette phase de l'autoconfrontation croisée (indiquant ainsi que le sujet est clôt). La forme hésitante et hachée, des phrases des deux professionnels, est un indice des difficultés à s'expliquer avec la situation et des émotions ressenties, en lien avec la «plurivocalité » intérieure qui fait face aux « équivoques du travail » (Clot, 2006, p. 165),

- Enfin, les deux points de vue sont bien partagés. Dans cet exemple, en particulier, Hervé semble bien selon les moments du dialogue, incarner l'un et l'autre des points de vue, Bertrand jouant lui, une partition différente puisqu'il devient clinicien à la place du clinicien. Ce constat n'infirme cependant pas notre hypothèse de partage, entre pairs, de

11 Position assez implicite mais qui nous semble être une toile de fond très présente dans les premières réactions d'Hervé, plutôt défensives.

12 Il est remarquable de constater, dans ce passage, la quasi absence du chercheur intervenant, puisque d'une certaine façon, Bertrand a pris sa place dans une forme de «nomadisme clinique ». 
deux termes opposés d'un même problème professionnel. Un masseur-kinésithérapeute doit fonder ses techniques sur des preuves scientifiques. Il doit aussi tenir compte de ses « éprouvés » empiriques.

Ainsi, pouvons-nous confirmer la présence d'un dilemme historique de métier, identifié grâce aux traces qu'il imprime aux dialogues entre professionnels du métier.

Au-delà de son intérêt pour une démarche méthodologique d'ingénierie de certification (pour la conception d'un diplôme professionnel) ou de gestion des ressources humaines (pour se doter d'un référentiel de compétences en entreprise), ce repérage permet de fournir aux professionnels engagés dans une co-analyse de leur travail, une forme d'objectivation des dilemmes qu'ils affrontent. Plusieurs expériences ont montré que les tentatives d'objectivation des pratiques professionnelles, y compris quand elles n'aboutissent pas totalement au plan de cette objectivation, sont de bons instruments cliniques de relance des dialogues entre professionnels (Biryukova, \& Bril, 2002 ; Fernandez, 2004 ; Simonet, 2011).

Nous avons pu, en effet, constater l'intérêt clinique d'accompagner la présentation d'un référentiel en dilemmes et acquis, d'extraits de dialogues sous forme de traces vidéo et écrites, qui rendent palpables, pour les professionnels, des « ressentis». Ils favorisent l'appropriation de ces éléments et invitent ainsi à la reprise du dialogue entre pairs, et à la controverse.

Sur la base de cette analyse, nous avons pu déterminer, avec les masseurs-kinésithérapeutes, une série de neuf dilemmes. Nous les avons présentés sous la forme d'un schéma où chaque dilemme est représenté par une formulation de ses deux termes, reliés par une flèche (voir Figure 1), dans l'objectif de rendre le concept parlant pour les membres du collectif initial de professionnels, mais aussi pour la direction de l'établissement, voire pour d'autres masseurskinésithérapeutes ne travaillant pas dans ce centre.

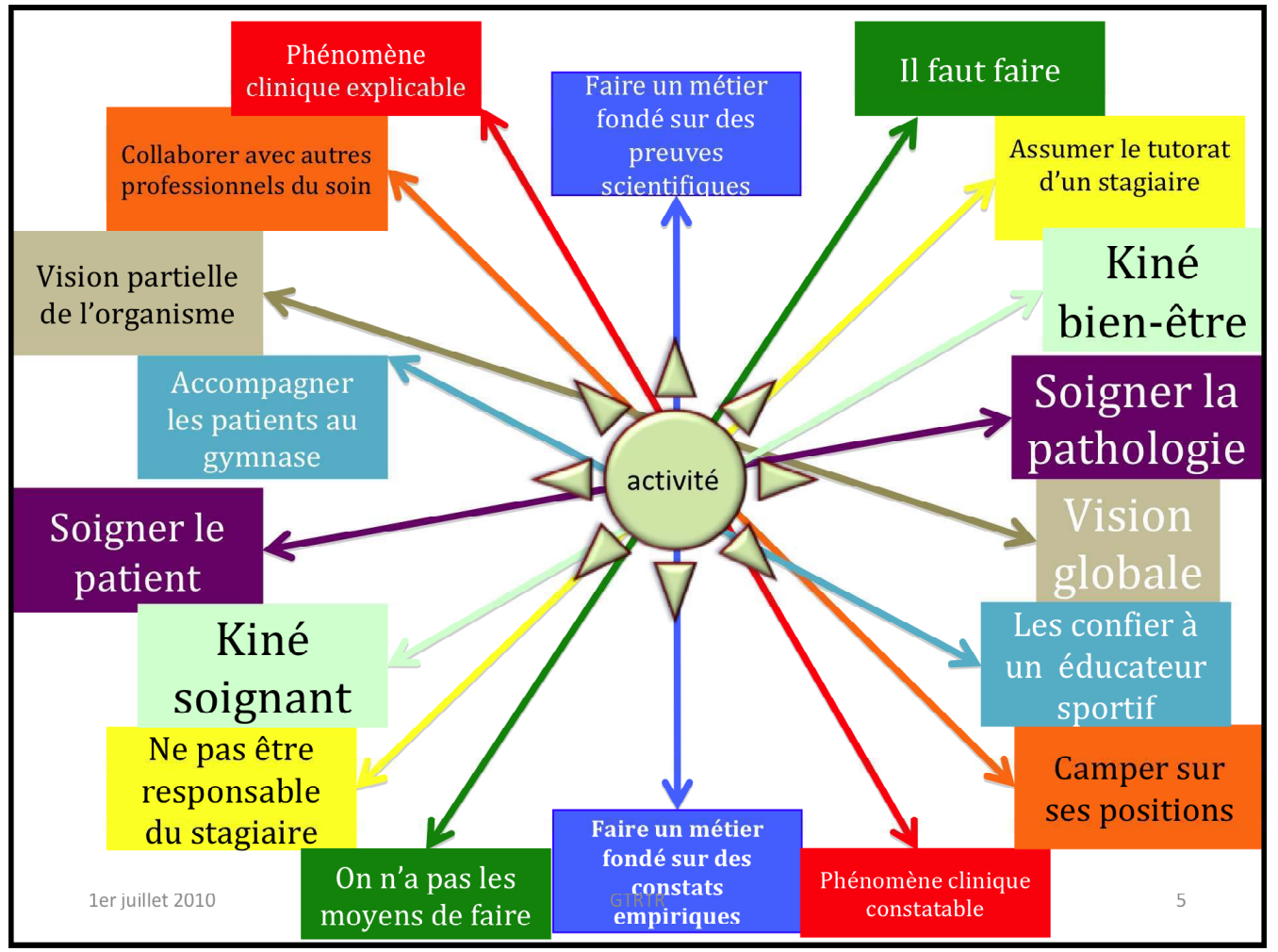

Figure 1 : Présentation schématique des 9 dilemmes identifiés dans les activités desmasseurs-kinésithérapeutes du centre de rééducation

Figure 1: Schematic presentation of 9 dilemmas identified in the activities of the physiotherapists at the rehabilitation center 
De même, cette intervention a permis d'identifier de très nombreux acquis, de nature très variée, mais dont la portée, plus ou moins singulière ou générique, a pu être révélée par une mise en débat, dans le groupe complet. Certains acquis, très spécifiques à une situation, ont été écartés car ne faisant pas référence pour le métier. D'autres ont été reformulés pour les faire «monter en généralité ». Pour les mêmes raisons de démonstration, ces acquis ont été présentés sous la forme d'un schéma (voir Figure 2) qui vise à affirmer, d'une part la diversité des acquis (par des couleurs différentes, des tailles de rectangles variables), mais aussi la non-correspondance directe entre un dilemme et un acquis.

En cela, nous avons pu nous appuyer sur l'expérience réalisée avec les syndicalistes-cadres (Clot, et al., 2009) où la présentation initiale des dilemmes et acquis dans un tableau à double entrée avait conduit à une mise en lien de ces entités, un peu simplificatrice. Dans la réalité, c'est parfois plusieurs acquis qui aident à gérer un dilemme. Parfois aussi, on peut identifier un acquis, par exemple une catachrèse, mais sans que le dilemme affronté n'apparaisse explicitement, à ce stade.

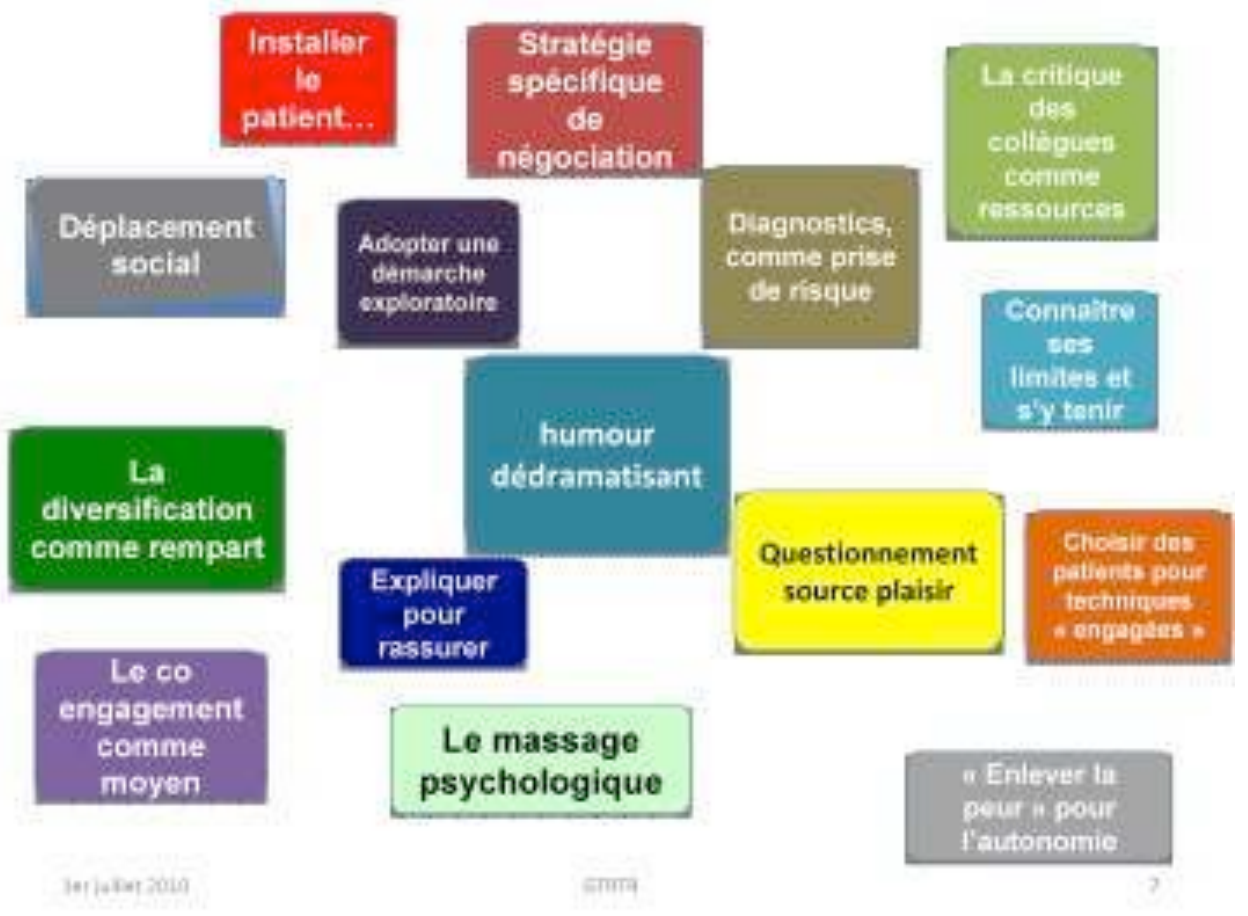

Figure 2 : Présentation schématique des 15 acquis d'expérience identifiés dans les activités des masseurs-kinésithérapeutes du centre de rééducation

Figure 2: Schematic presentation of 15 pieces of acquired knowledge identified in the activities of the physiotherapists at the rehabilitation center.

Ce référentiel, ainsi construit, a été présenté à plusieurs occasions à des groupes de masseurs-kinésithérapeutes, au-delà du groupe initial constitué pour l'intervention. L'effet dynamogène sur les dialogues professionnels a été immédiatement constatable dans tous les cas. Ce référentiel en dilemme et acquis «parle» aux professionnels et leur donne un bon prétexte pour parler de leur métier ensemble.

Si le faible nombre d'expérimentations impose d'être prudent, il semble que cette forme de référentialisation, qui tente de décrire un métier en se focalisant sur ses irrésolus, est donc un bon instrument de reprise des dialogues entre professionnels sur leur travail et, par là, un bon moyen d'intervention en clinique du travail. Mais, comment peut-on expliquer cette « efficacité » clinique que nous soupçonnons? 


\section{4.- Référentiel et catégorisation implicite}

Avant de répondre à cette question, revenons sur un constat fait au cours d'autres interventions avec des professeurs de sport ou des entraîneurs sportifs de haut niveau (Balas, 2005,2008 ) et qui s'est répété avec les masseurs-kinésithérapeutes, comme nous pouvons l'observer dans l'extrait reproduit dans le tableau 1. Il s'agit de l'utilisation répétitive d'expressions, par les professionnels engagés dans ces interventions, telles que «ça dépend » en réponse à une question sur leur travail.

Ce type de réponse à caractère idiosyncrasique, nous a semblé, dans un premier temps, purement défensif. Dans une démarche clinique, ces réponses nous semblaient constituer des «dérobades », des manières de ne pas s'engager pleinement dans l'analyse et ainsi de conduire le dialogue à l'impasse ${ }^{13}$. Pourtant, la répétition de ces expressions, y compris par des professionnels très impliqués et dans des séquences dialogiques très vives, nous a conduit à formuler une autre hypothèse, d'autant que d'autres traces d'interventions disponibles font apparaitre les mêmes expressions répétitives (par exemple Prot, \& ReilleBaudrin, 2007, p. 56).

Si les professionnels, parfois, utilisent ce type d'expression, c'est aussi parce que la réponse à la question posée est réellement conditionnée par une diversité de facteurs : ça dépend, en effet, du contexte, des collègues, de la météo, du patient... mais aussi des styles de chacun, qui, comme le montrent les travaux de Clot et Faïta déjà cités (2000) ne s'opposent pas à l'expression générique du métier, mais l'attaque pour l'enrichir.

Ainsi, quand un professionnel dit «ça dépend» ou «des fois », il exprime la diversité du patrimoine, à l'intérieur du métier, y compris en faisant appel à des «figures » passées d'anciens collègues devenus des références pour chacun. Ces expressions doivent alors être perçues, par le clinicien, non comme défensives, mais plutôt comme l'expression des traces historiques et développementales du métier d'appartenance du professionnel.

Dans cette voie, nous avons alors pu développer encore notre point de vue sur ces réponses récurrentes des professionnels et y voir les traces d'un «proto-référentiel », forme de « référentiel opératif commun» (Giboin, 2004). Ce proto-référentiel organise l'action des professionnels du métier, de manière implicite la plupart du temps, mais cependant avec une permanence qui signe sa participation à la définition du genre professionnel. Être un professionnel expérimenté du métier passe par l'appropriation de cette «grille» de lecture de la diversité des situations qui peut parfois apparaitre comme le rébus évoqué par Bruner (1996, p. 190). S'il est difficile de déchiffrer ce rébus, il est encore plus difficile de se retrouver seul «face à l'étendue des bêtises possibles » (Darré, 1994). C'est « une sorte d'intercalaire social, un corps d'évaluation partagée qui règle l'activité personnelle de façon tacite » (Clot, et al., p. 19) et fixe aussi les variantes acceptables et celles qui ne le sont pas pour un membre du métier.

Ainsi, quand on intervient avec un groupe de professionnels et que l'on sollicite de leur part un effort de description détaillée de ce qu'est leur métier, de ce qui le caractérise et donc fixe la frontière entre ce qui est «dans le métier» de ce qui n'y est pas, alors, ces opérateurs entreprennent une démarche taxonomique. En effet, pour décrire un tout, il est nécessaire d'organiser ce tout en sous-parties, en catégories cohérentes. Ce faisant, les professionnels retrouvent, de manière incidente, le proto-référentiel évoqué. L'effet est alors immédiat puisqu'il conduit les professionnels à « dénaturaliser » leurs activités (Fernandez, 2009) et à redécouvrir leur polyphonie sociale dans des échanges avec leurs collègues. Au cours des dialogues réglés, permis par l'organisation d'un cadre méthodologique, ces professionnels revivent, en conscience et en "accéléré » le parcours de leur professionnalisation. Le rébus

13 On peut d'ailleurs analyser avec intérêt l'effet affectif produit, sur le clinicien, par de tels épisodes (Scheller, 2002). 
auquel on peut assimiler le référentiel «interne » au métier est alors déchiffré et mis en visibilité par le processus de rédaction d'un référentiel « externe ».

S'il est illusoire de penser que le référentiel externe pourrait être une copie conforme du référentiel interne, on peut penser que comme dans le rapport entre la pensée et le langage, documenté par Vygotski (1934/1997), l'expression externe du référentiel trahit sa forme interne, aux deux sens du terme.

Dans notre exemple avec les masseurs-kinésithérapeutes du centre de rééducation, l'entretien d'autoconfrontation croisée entre Bertrand et Hervé a duré environ une heure et trente minutes. Il a donné lieu à plus de mille tours de parole. Cette retranscription fait apparaitre pas moins de cent-trente-cinq occurrences des expressions «ça dépend », « ou alors », « des fois» ou «en fait». Ce premier repérage confirme un «sentiment» éprouvé lors du déroulement de l'intervention et déjà perçu précédemment. Les locutions qui semblent exprimer une singularité de son auteur, ou de la situation rencontrée, sont si fréquentes qu'elles révèlent un arrière-plan générique, implicite, mais structurant pour chaque professionnel du métier.

Les processus de référentialisation, parce qu'ils mettent au jour ces cadres implicites sont alors des instruments efficaces d'intervention qui permettent aux professionnels associés de reprendre en main le destin de leur métier et ainsi de développer leur pouvoir d'agir (Clot, 2008a).

On peut même alors dire que cet effet local, parce qu'il débouche sur la rédaction d'un référentiel descriptif de métier, et à la condition que ce dernier reste discutable ${ }^{14}$, offre alors un instrument de propagation de ces effets, en confiant à des cercles de plus en plus élargis de professionnels, le soin de débattre avec le référentiel. En effet, ce référentiel, puisqu'il renoue avec un cadre implicite générique du métier, permet bien de dépasser l'action locale et de généraliser ses effets à des professionnels éloignés de sa conception. Nous venons d'ailleurs d'être sollicités par une revue professionnelle pour produire un article revenant sur cette expérience. Nous voyons dans ce type de demande un élément favorable à l'organisation des conditions sociales de cette « rencontre », ce qui relève du dialogue social. On peut cependant observer à travers cet exemple, comment activité scientifique et intervention «sociale» peuvent s'étayer mutuellement.

\section{Pour conclure}

Vygotski, dans son ouvrage "pensée et langage », indiquait que «c'est seulement dans la dispute, dans la discussion qu'émergent les éléments fonctionnels qui déclenchent le développement de la réflexion » (1934/1997, p. 105). C'est avec cette orientation que nous avons abordé l'épineuse question de la formalisation de l'expérience professionnelle singulière, dans un outil générique.

Cependant au lieu de chercher à traduire cette expérience par une formulation la plus authentique possible, pour ensuite produire une norme générique, nous avons voulu que cette expérience, une fois décrite dans un référentiel, permette de poursuivre la «dispute » entre professionnels, ou entre usagers (enseignants et formateurs, jury...), (Balas, 2012).

Nous avons parié qu'un référentiel qui favorise la dispute entre ses utilisateurs permet de poursuivre la réflexion sur les objets qu'il décrit, l'activité, le métier, sa transmission... Pour favoriser cette dispute, nous avons dû produire un document non fini, et perpétuellement à entretenir, en répertoriant certains des irrésolus du métier.

14 Ce qui impose des conditions techniques, que nous avons évoquées en proposant une éventuelle solution, mais aussi sociales tant il est vrai que les professionnels concernés sont rarement invités à «l'entretien » de « leur» référentiel. 
Et alors, ce référentiel s'est, en effet, avéré un bon moyen de relance du dialogue entre ses utilisateurs, comme nous en rendons compte par ailleurs (Balas, 2011c). Nous avons alors voulu nous interroger sur les ressorts de cette «efficacité » clinique. Il apparait que le processus de conception, puis l'usage de ces référentiels descriptifs, parce qu'ils obligent les professionnels à parcourir à nouveau le trajet de leur professionnalisation, de manière accélérée et explicite, leur font redécouvrir une «grille» implicite qui organise leur activité en catégorisant les situations affrontées. Mieux encore, cette grille implicite, parce qu'elle est explicitée dans les dialogues, s'en trouve modifiée.

Ce constat amène alors à penser que les interventions cliniques que nous conduisons habituellement avec des professionnels, dans le cadre d'une clinique de l'activité et qui visent à accompagner les professionnels dans la reprise en main de l'histoire de leur métier pourraient trouver, dans les processus de référentialisation, un moyen «technique » complémentaire. En effet, dans les processus plus habituels d'intervention en clinique de l'activité, si l'on peut imaginer des «habitudes» dans ce cadre, ce que l'on vise est le développement des activités de travail des personnes, développement qui produit des effets positifs sur la santé des individus et du collectif (Clot, 2010 ; Fernandez, 2009).

Dans notre proposition, la co-conception d'un référentiel descriptif du métier, ce que l'on désigne par un référentialisation, produit, de même, un effet de développement du métier décrit. Dans les deux cas, c'est bien par le biais de ce développement, que l'on peut juger de la dimension « intervenante » des processus cliniques conduits.

Le constat de cette dimension intervenante des processus de référentialisation permet alors légitimement de réaffirmer l'importance d'associer des professionnels du métier aux travaux de création ou de rénovation des référentiels des diplômes professionnels, car ces processus sont agissants sur le métier décrit et pas seulement un phénomène expert extérieur.

\section{BIBLIOGRAPHIE}

Balas, S. (2005). Analyse des conditions de développement d'un genre professionnel chez les conseillers d'animation sportive des services déconcentrés du ministère de la jeunesse, des sports et de la vie associative. Mémoire en vue de l'obtention du diplôme de l'Insep. Paris: INSEP.

Balas, S. (2008). Le métier d'entraîneur au travail. Comment co-construire un cadre d'analyse développementale avec les professionnels de l'entraînement sportif ? In Cahiers de l'INSEP n³9 : Actes des entretiens de l'INSEP des 11 et 12 octobre 2007. Paris: INSEP éditions, pp. 321-328.

Balas, S. (2011a). Kinésithérapeute, un métier de référence. Nouvelle revue de psychosociologie, $\mathrm{n}^{\circ} 12$, 2011/2, 223-238.

Balas, S. (2011b). Le référentiel, un outil de formation, un instrument de développement du métier. Le métier de masseur-kinésithérapeute en référence. Thèse pour le doctorat de sciences de l'éducation. Paris: CNAM.

Balas, S. (2011c). Le référentiel des masseurs-kinésithérapeutes : l'usage comme norme. "Université et métiers de la formation : quels enjeux, quelles spécificités? ", Colloque du réseau des Universités préparant aux Métiers de la Formation, Avignon, 12 et 13 mai 2011.

Balas, S. (2012). Transmettre un métier? L'exemple des masseurs-kinésithérapeutes. Biennale internationale : Éducation - Formation-Pratiques professionnelles, 3 au 6 juillet 2012, Paris: CNAM.

Bernstein, N. A. (1996). About dexterity and its development. In M. L. Latash \& M. T. Turvey (Eds.), Dexterity and its development, with On dexterity and its development by Bernstein N.A. (pp. 1244). Mahwah New Jersey: Lauwrence Erlbaum Associates.

Biryukova, E., \& Bril, B. (2002). Bernstein et le geste technique. In B. Bril \& V. Roux (Eds.) Le geste technique. Réflexions méthodologiques et anthropologiques. Ramonville Saint-Agne: Eres.

Bournel Bosson, M. (2005). Les organisateurs du mouvement dialogique : Autoconfrontations croisées et activité des conseillers en bilans de compétences. Thèse pour le doctorat de psychologie. Paris: CNAM.

Bruner, J. (1996). L'Éducation, entrée dans la culture, Paris: Retz. 
Chauvigné, C. (2010). Les référentiels en formation, in C. Chauvigné \& Y. Lenoir, (Sous la coord.). Les référentiels en formation : enjeux, légitimité, contenu et usage, Recherche \& Formation, $\mathrm{N}^{\circ}$ 64-2010, pp. 77-89

Clot, Y. (1995). Le travail sans l'homme? Pour une psychologie des milieux du travail et de vie. Paris : La découverte.

Clot, Y. (2004). Action et connaissance en clinique de 1'activité. @ ctivités, 1(1), http://www .activites.org/v1n1/clot.pdf

Clot, Y. (2005). L'autoconfrontation croisée en analyse du travail : l'apport de la théorie bakhtinienne du dialogue. In L. Filliettaz \& J.-P. Bronckart (Eds.), L'analyse des actions et des discours en situation de travail. Concepts, méthodes et applications (pp. 57-75). Louvain-la-Neuve: Peeters.

Clot, Y. (2006). Clinique du travail et clinique de l'activité. Nouvelle revue de psychosociologie, 2006/1(1), 165-177.

Clot, Y. (2008a). Travail et pouvoir d'agir. Paris: PUF.

Clot, Y. (2008b). La recherche fondamentale de terrain : une troisième voie. Éducation Permanente, 2008/4(177), 67-77.

Clot, Y. (2010). Le travail à cour. Pour en finir avec les risques psychosociaux. Paris: La découverte.

Clot, Y., \& Faïta, D. (2000). Genres et styles en analyse du travail. Concepts et méthodes. Travailler, 4, 7-42.

Clot, Y., Faïta, D., Fernandez, G., \& Scheller, L. (2001). Entretiens en auto confrontation croisée : une méthode en clinique de l'activité, Éducation Permanente, 2001/1(146), 17-25.

Clot, Y., \& Gori, R. (2003). Catachrèse: éloge du détournement. Nancy: PUN

Clot, Y., \& Leplat, J. (2005). La méthode clinique en ergonomie et en psychologie du travail. Le Travail Humain, 68(4), 289-316.

Clot, Y., Litim, M., Prot, B., \& Zittoun, M. (2008). Éducation à la conduite et à la sécurité routière : les acquis de l'expérience. Rapport, Direction de la Sécurité et la Conduite Routières, Ministère du développement durable.

Clot, Y., Tomás, J.-L., \& Kloetzer L. (2009). Du travail syndical au référentiel. La VAE à la Confédération Française de l'Encadrement - Confédération Générale des Cadres. Rapport de recherche CNAM, CRTD

Darré, J.-P. (1994). Le mouvement des normes avec Bakhtine et quelques agriculteurs. J.-P. Darré (Ed.), Pairs et experts dans l'agriculture (pp. 15-29). Toulouse: Erès.

Fernandez, G. (2004). Développement d'un geste technique. Histoire du freinage en gare du nord. Paris: CNAM. Thèse pour le doctorat de psychologie. Paris: CNAM.

Fernandez, G. (2009). Soigner le travail. Itinéraire d'un médecin du travail. Paris: Eres.

Figari, G. (1994). Evaluer : quel référentiel? Bruxelles: De Boeck Université.

Giboin, A. (2004). La construction des référentiels communs dans le travail coopératif. In J.M. Hoc, \& F. Darses (Eds.). Psychologie ergonomique : tendances actuelles (pp. 119-139). Paris: PUF.

Kostulski, K., Clot, Y., Litim, M., \& Plateau, S. (2011). L'horizon incertain de la transformation en clinique de l'activité : une intervention dans le champ de l'éducation surveillée. Activités, 8(1), 129-145, http://www .activites.org/v8n1/v8n1.pdf

Maillard, F. (Sous la dir.) (2008). Diplômes et certifications professionnelles. Nouvelles normes et nouveaux enjeux. Rennes: Presses Universitaires de Rennes

Olry, P., \& Vidal-Gomel, C. (2011). Conception de formation professionnelle continue : tensions croisées et apports de l'ergonomie, de la didactique professionnelle et des pratiques d'ingénierie. Activités, 8(2), 115-149, http://www .activites.org/v8n2/v8n2.pdf

Ombredane, A., \& Faverge, J.-M. (1955). L'Analyse du travail. Facteur d'économie humaine et de productivité. Paris: PUF

Pastré, P. (2011). La didactique professionnelle. Approche anthropologique du développement chez les adultes. Paris: PUF

Prot, B. (2007). Expérience et référentiel : Le problème de Galilée. In F. Neyrat, (sous la dir.). La validation des acquis de l'expérience. Reconnaissance d'un nouveau droit. Bellecombes-enBauges : éditions du croquant, pp. 199-218. 
Prot, B. (2011). Apprentissage de la conduite et sécurité routière : Un dilemme de référence pour la conception d'un référentiel de diplôme d'enseignant. Activités, 8(2), 189-201, http://www .activites.org/v8n2/v8n2.pdf

Prot, B. \& Reille-Baudrin, E. (2007). Entre le référentiel et l'activité : le problème de la prospective du métier. Une étude avec des employés de bureau. CPC documents, 2007-1.

Prot, B., Ouvrier-Bonnaz, R., Mezza, J., Reille-Baudrin, E., \& Verillon, P. (2010). Les dilemmes d'activités. Pour une approche clinique des correspondances entre travail et formation professionnelle. Recherche et formation, $\mathrm{n}^{\circ} 63-2010,63-76$.

Rabardel, P. (2002, 2ème édition). Le langage comme instrument ? Éléments pour une théorie instrumentale élargie. In Y. Clot, Avec Vygotski (pp. 265-289).Paris: La Dispute,

Rabardel, P. (2005). Instrument, activité et développement du pouvoir d'agir. P. Lorino, \& R. Teulier (Ed.). Entre connaissance et organisation : l'activité collective (pp. 251-265). Colloque de Cerisy. Paris: La découverte,

Rabardel, P., \& Pastre, P. (Eds.) (2005). Modèle du sujet dans la conception. Dialectiques activités développement. Toulouse: Octarès

Samurçay, R., \& Rabardel, P. (2004). Modèles pour l'analyse de l'activité et des compétences. Propositions. Dans : R. Samurçay, \& P. Pastré (Eds.). Recherches en didactique professionnelle (pp. 163-180). Toulouse: Octarès,

Scheller, L. (2002). Clinique de l'activité, haine, travail. Cliniques méditerranéennes, 2002/2(66), 85103.

Simonet, P., Caroly, S., \& Clot, Y. (2011). Méthodes d'observation de l'activité de travail et prévention durable des TMS : action et discussion interdisciplinaire entre clinique de l'activité et ergonomie. Activités 8(1), 104-128, http://www .activites.org/v8n1/v8n1.pdf

Simonet, P. (2011). L'hypo-socialisation du mouvement : prévention durable des troubles musculosquelettiques chez des fossoyeurs municipaux. Thèse pour le doctorat de psychologie. Paris: CNAM.

Sorel, M., \& Wittorski, R. (2005). La professionnalisation en actes et en questions. Paris: L'Harmattan

Tomàs, J.-L. (2007). Les conflits de critères en haltérophilie : sources et ressources du développement de l'activité d'analyse. Psychologie de l'interaction, 11-39.

Verillon, P., \& Rabardel, P. (1995). Cognition and artefact: a contribution to the study of thought in relation to intrumented activity. European journal of psychology of éducation, 10(3), 77-101

Vygotski, L. (1925/2005, traduction de F. Sève). Psychologie de l'art. Paris: La dispute.

Vygotski, L. (1934/1997, traduction F. Sève). Pensée et langage. Paris: La Dispute.

Vygotski, L. (2003, traduction F. Sève et G. Fernandez). Conscience, inconscience, émotions. Paris : La Dispute.

Vygotski, L. (2011, traduction O. Anokhina, F. Sève, \& G. Fernandez). Leçons de psychologie. Paris: La dispute.

\section{RESUME}

La conception d'un référentiel descriptif d'un métier, à des fins de formation, ou de management, produit un effet sur l'activité des personnes associées au processus. Une fois rédigé, ce référentiel devient cependant un document normalisant et fixiste, peu en rapport avec la dynamique de l'activité de travail. Avec un collectif de masseurs-kinésithérapeutes, nous avons réalisé une intervention clinique qui visait à répertorier les irrésolus de leur métier, qui nous semblent un accès possible au réel de l'activité. Parmi ces irrésolus, certains sont particulièrement pérennes et sont caractéristiques de l'exercice du métier: ce sont les dilemmes qui confrontent les professionnels, dans l'action, à des choix impossibles entre deux ou plusieurs solutions insatisfaisantes. Face à ces obstacles insolubles, pour agir malgré tout, ces opérateurs développent leur expérience, 
dont les acquis sont capitalisables.

Sur cette base, notre intervention en clinique de l'activité avec les masseurs-kinésithérapeutes, a permis de concevoir un référentiel en dilemme et acquis qui favorise, à l'usage, la relance des dialogues entre professionnels. Son efficacité clinique semble s'expliquer par la présence d'un «proto-référentiel », instrument générique de préorganisation implicite des situations affrontées, et que le processus de référentialisation permet de redécouvrir et de remettre en débat. Ainsi, le cadre artificiel de l'intervention clinique permet de requestionner le cadre écologique sous-jacent du métier.

\section{MOTS CLES}

Activité, Masseurs-kinésithérapeutes, référentiel, clinique, dilemme

\section{REFERENCEMENT}

Balas, S. (2013). Intervenir avec des masseurs-kinésithérapeutes: le développement en référence. Activités, 10(1), 93-111, http://www.activites.org/v10n1/v10n1.pdf

Article soumis le 3 janvier 2012, accepté pour publication le 3 janvier 2013 